\title{
Resolution of Microscopic Protonation Mechanisms in Polyprotic Molecules
}

\author{
Michal Borkovec ${ }^{*}$, Marcin Brynda ${ }^{\mathrm{a}}$, Ger J. M. Koper ${ }^{\mathrm{b}}$, and Bernard Spiess ${ }^{\mathrm{c}}$
}

\begin{abstract}
Microscopic ionization equilibria can be fully resolved by means of a novel site-binding model, which is based on a cluster expansion technique borrowed from statistical mechanics. This model permits a parameterization of the problem, and offers substantial advantages over the commonly used microscopic equilibrium constants. While the number of microconstants grows very rapidly with the size of the molecules, the necessary number of parameters of the site-binding model remain small, and one can even obtain common sets of such parameters within a homologous series of molecules. Based on this approach, two methods to obtain such microconstants are discussed, namely based on NMR titration data, and on the analysis of potentiometric titrations within a homologous series.
\end{abstract}

Keywords: Acidity constants · Macroconstants $\cdot$ Microconstants $\cdot$ NMR $\cdot$ Potentiometric titration

\section{Introduction}

The understanding of protonation mechanisms of polyprotic molecules represents not only an important topic in analytical chemistry, but is equally relevant in several applied disciplines, such as biochemistry, pharmacy, or environmental chemistry [1-3]. Classical potentiometric techniques yield information primarily about the macroscopic protonation equilibria, which only specify the number of protons bound without reference to the protonation state of the ionizable sites. To obtain direct insight into the protonation mechanism of a molecule, one needs information about the microscopic equilibria, where the protonation state of each individual site is considered. From the latter, one can assess the properties of individual ionizable

\footnotetext{
${ }^{\star}$ Correspondence: Prof. M. Borkovec

Department of Inorganic, Analytical, and Applied

Chemistry

University of Geneva

30 Quai Ernest-Ansermet

$\mathrm{CH}-1211$ Geneva 4

Tel.: +4122 7026405

Fax: +4122 7026069

E-Mail: michal.borkovec@cabe.unige.ch

aDepartment of Chemistry

University of California, Davis

California 95616, USA

bLaboratory of Physical Chemistry

Delft University of Technology

Julianalaan 136

NL-2628 BL Delft, The Netherlands

'Laboratory of Molecular Pharmacochemistry

UMR 7081 CNRS-ULP

Faculty of Pharmacy, BP 24

67401 Illkirch Cedex, France
}

residues, such as the degree of protonation (i.e. site-specific titration curves) and the microscopic ionization constants (i.e. microconstants). To discuss these aspects concerning the microscopic ionization mechanisms in polyprotic molecules, the corresponding microscopic equilibria must be resolved completely.

Initially, the determination of microscopic ionization constants was approached with optical spectroscopy and with comparative studies of similar compounds [4][5]. However, these techniques were hardly satisfactory, as optical spectra of polyprotic molecules are notoriously difficult to analyze. The situation has substantially improved with the availability of high-resolution and multi-dimensional nuclear magnetic resonance (NMR) techniques [6]. Due to the fact that proton exchange is sufficiently fast, the chemical shift often is a linear function of the degree of protonation of the neighboring ionizable site. Thus, by performing NMR titrations, and plotting chemical shift as a function of $\mathrm{pH}$, one can obtain the degree of protonation of individual ionizable sites in a polyprotic molecule [3][7-10].

However, even with this kind of information at hand, the evaluation of the microscopic ionization constants is non-trivial. Microscopic ionization equilibria of polyprotic molecules are usually parameterized in terms of all possible microconstants, and as the number of ionizable sites increases, their number becomes quickly overwhelming. Already for a simple triprotic molecule, twelve microconstants have to be determined - this problem was recently successfully tackled for several triphosphates with ${ }^{31} \mathrm{P}-\mathrm{NMR}$ [7-9]. In the general case of $N$ ionizable sites, there are $N 2^{N-1}$ unknown microconstants [11]. In spite of the availability of individual site-titration curves from NMR, it is indeed not obvious how to determine all such constants for larger molecules.

The aim of this short article is to summarize the significant progress that can be made in the resolution of microscopic ionization equilibria of polyprotic molecules by applying so-called cluster expansion techniques borrowed from statistical mechanics [12]. The basic idea is to perform a cluster expansion of the free energy of the microstates [11], which provides an appropriate parameterization of the problem, and reduces the number of unknowns dramatically. The description can not only be systematically improved by including higher order contributions, but also incorporates inherent molecular symmetries, which are usually not taken into account in the classical description of microscopic equilibria. We present two different approaches for the resolution of microscopic equilibria in polyprotic molecules. First, the analysis is based on site-specific NMR titration data [13]. Second, by analyzing homologous series of molecules, we show that microscopic equilibria can be equally resolved based on classical potentiometric titration data alone. 


\section{Statistical Mechanics of Protonation Equilibria}

The protonation state of a polyprotic molecule can be specified by introducing a two-valued state variable $s_{i}$ for each individual site $i(i=1,2, \ldots, N)$ such that $s_{i}=1$ if the site is protonated and $s_{i}=0$ if the site is deprotonated. The protonation microstate is then uniquely defined by the set of state variables $\left\{s_{1}, s_{2}, \ldots, s_{N}\right\}$, abbreviated as $\left\{s_{i}\right\}$. To each microstate one can assign a standard free energy of formation $F\left(\left\{s_{i}\right\}\right)$ with respect to the fully deprotonated state and for unit activity of protons. The free energy can be parameterized through the coefficients of the cluster expansion [3][11]

$$
\frac{\beta F\left(\left\{s_{i}\right\}\right)}{\ln 10}=-\sum_{i} \mathrm{p} \hat{K}_{i} s_{i}+\frac{1}{2 !} \sum_{i, j} \epsilon_{i j} s_{i} s_{j}+\frac{1}{3 !} \sum_{i, j, k} \lambda_{i j k} s_{i} s_{j} s_{k}+\ldots
$$

where the sums run over all the sites, $\beta^{-1}$ is the thermal energy, $\mathrm{p} \hat{K}_{i}$ is the microscopic ionization constant of the site $i$ given all other sites are deprotonated, and $\varepsilon_{i j}$ and $\lambda_{i j k}$ are pair and triplet interaction parameters (or interactivity parameters [7]). The pair interactions obey the symmetry relation $\varepsilon_{i j}$ $=\varepsilon_{j i}$, and without loss of generality one can set $\varepsilon_{i i}=0$. For the triplet interactions one similarly has $\lambda_{i j k}=\lambda_{j i k}=\lambda_{i k j}$ and $\lambda_{i i j}=\lambda_{i j i}=$ $\lambda_{i j j}=0$. Higher order clusters can also be included if necessary. As we shall see, however, the cluster expansion converges rapidly, and in many situations one can terminate the expansion already at the pair interaction level.

The magnitude of the interactions decreases quickly with increasing distance between the ionizable groups, and interactions beyond the nearest neighbors can be usually neglected [3][11]. Molecular symmetry can be used to further reduce the number of independent parameters. Thus, the microscopic equilibria can be parameterized by specifying the microconstants $\mathrm{p} \hat{K}_{i}$ and the interaction parameters $\varepsilon_{i j}$ (and eventually $\lambda_{i j k}$. These cluster parameters fully define the microscopic equilibria, and their number can be moderate even for a complex molecule.

The probability of a given microstate can be now written as [3][11]

$$
p\left(\left\{s_{i}\right\}\right)=\Xi^{-1} a_{\mathrm{H}}^{n} e^{-\beta F\left(\left\{s_{i}\right\}\right)}
$$

where we have introduced the activity of protons $a_{\mathrm{H}}$ (where $\mathrm{pH}=-\log _{10} a_{\mathrm{H}}$ ), the total number of bound protons

$$
n=\sum_{i=1}^{N} s_{i}
$$

and a normalization constant

$$
\Xi=\sum_{\left\{s_{i}\right\}} a_{\mathbf{H}}^{n} e^{-\beta r^{\prime}\left(\left\{s_{i}\right\}\right)}
$$

This normalization constant can be interpreted as a partition function.

The average degree of protonation of an individual site $m$ can be evaluated by averaging the state variable $s_{m}$ over all microstates, namely

$$
\theta_{m}=\sum_{\left\{s_{i}\right\}} s_{m} p\left(\left\{s_{i}\right\}\right)
$$

These site-specific titration curves can be directly measured by NMR.

The average degree of protonation of the entire molecule is simply the average of all site-specific titration curves, namely

$$
\theta=\frac{1}{N} \sum_{m=1}^{N} \theta_{m}
$$

This macroscopic titration curve can be measured by classical potentiometry.

The above expression can be rewritten in terms of more familiar quantities by realizing that the partition function $\Xi$ given in Eqn. 4 can be expressed as

$$
\Xi=\sum_{n=0}^{N} \bar{K}_{n} a_{\mathrm{H}}^{n}
$$

where $\bar{K}_{n}$ are the cumulative association constant. The commonly used macroscopic step-wise dissociation constants can be expressed in terms of the cumulative constants as p $K_{n}=\log _{10} \bar{K}_{n} / \bar{K}_{n-1}$. Eqn. 7 is also referred to as the binding polynomial [1]. We can introduce the probability of a particular macrostate as

$$
P_{n}\left(a_{\mathrm{H}}\right)=\Xi^{-1} \bar{K}_{n} a_{\mathrm{H}}^{n}
$$

and the macroscopic titration curve can be expressed as

$$
\theta=\frac{a_{\mathrm{H}}}{N} \frac{\partial \log \Xi}{\partial a_{\mathrm{H}}}=\frac{1}{N} \sum_{n=0}^{N} n P_{n}\left(a_{\mathrm{H}}\right)
$$

or equivalently [1][2]

$$
\theta=\frac{1}{N} \frac{\sum_{n=0}^{N} n \bar{K}_{n} a_{\mathrm{H}}^{n}}{\sum_{n=0}^{N} \bar{K}_{n} a_{\mathrm{H}}^{n}}
$$

From this function one can determine all cumulative association constants (or equivalently all macroscopic $\mathrm{p} K$ values).

Additional quantities are of interest. The conditional probability to find a particular microstate within its macrostate $n$ can be introduced by splitting the microstate probabilities into a product

$$
p\left(\left\{s_{i}\right\}\right)=\pi_{n}\left(\left\{s_{i}\right\}\right) P_{n}\left(a_{H}\right) .
$$

Thereby, we have used the macrostate probability $P_{n}\left(a_{\mathrm{H}}\right)$ introduced in Eqn. 8 and $\pi_{n}\left(\left\{s_{i}\right\}\right)$ denotes the conditional microstate probability. Note that the macrostate probabilities are $\mathrm{pH}$ dependent, while the conditional microstate probabilities are constants. The latter can be simply expressed as

$$
\pi_{n}\left(\left\{s_{i}\right\}\right)=\bar{K}_{n}^{-1} e^{-\beta F\left(\left\{s_{i}\right\}\right)}
$$

All microconstants can be calculated in a straightforward fashion as well. These ionization constants commonly refer to the protonation reaction, where one particular site is being protonated. If we label this site with $j$, the association equilibrium can be written as

$$
\mathrm{A}\left\{s_{i}\right\}+\mathrm{H} \rightleftharpoons \mathrm{A}\left\{s_{i}^{\prime}\right\}
$$

where $s_{i}=s_{i}^{\prime}$ for all $i \neq j$ but $s_{j}=0$ and $s_{j}^{\prime}=1$. Using the free energy (Eqn. 1), the microscopic $\mathrm{p} K$ value for the reaction given by Eqn. 13 follows as [11]

$p \hat{K}_{\mathrm{A}\left\{s_{i}\right\}}=p \hat{K}_{j}-\sum_{k} \epsilon_{j k} \cdot s_{k}-\frac{1}{2} \sum_{k \cdot l} \lambda_{j k l} s_{k} \cdot s_{l}-\ldots$ (14)

When the triplet contributions are neglected, this relation reflects the group additivity concept for the estimation of ionization constants [14]. In the present approach, the microconstants represent secondary parameters, which can be easily evaluated once the primary cluster parameters are known.

\section{Microscopic Equilibria from NMR Titrations}

In favorable situations, one can measure the site-specific titration curves directly with NMR. This technique is most powerful when a $1 / 2$ spin nucleus is situated in 
close proximity to the ionizable group in question, and the other ionizable groups relatively far apart. The situation is almost optimally realized for phosphate and for amine groups. The protonation state of a phosphate group can be probed through the chemical shift of the corresponding ${ }^{31} \mathrm{P}$-nucleus, while for an amine group the ${ }^{15} \mathrm{~N}$-nucleus can be used. Due to the low natural abundance of the latter, the signals are weak, but can be enhanced with ${ }^{15} \mathrm{~N}$ enriched samples. In several situations, one can also use ${ }^{1} \mathrm{H}$ and ${ }^{13} \mathrm{C}$-NMR, but since these nuclei may not be in close proximity to the ionizable group of interest, crosscoupling effects may complicate the picture [13].

To obtain the site-specific titration curves, one simply measures the chemical shift of the proximal nucleus as a function of $\mathrm{pH}$. Since proton exchange is sufficiently fast, the degree of protonation of the site $m$ is then obtained from the corresponding chemical shift $\delta_{m}$ as [6]

$$
\theta_{m}=\frac{\delta_{m}-\delta_{m}^{(\text {dep })}}{\delta_{m}^{(\text {pro })}-\delta_{m}^{(\text {dep })}}
$$

where $\delta_{m}^{(\mathrm{pro})}$ and $\delta_{m}^{\text {(dep) }}$ are the corresponding chemical shifts in the fully protonated and deprotonated molecule.

Once these site-specific titration curves are known, they can be interpreted with the site-binding model discussed above. Practically, one uses a least-squares fitting procedure to extract the cluster parameters. In many situations their determination is unambiguous, but given the inherent noise in the data, some of the parameters sometimes cannot be properly determined. Once one has agreed on a set of cluster parameters, one can calculate all microconstants (as well as all macroconstants). Evaluating the $\mathrm{pH}$ dependent macrostate probabilities, and the $\mathrm{pH}$ independent conditional microstate probabilities, the microscopic protonation mechanism of the molecule can be visualized.

Let us illustrate the procedure with two inositol-phosphate analogues, namely 6-deoxy-6-fluoro-myo-inositol 1,4,5-tris (phosphate) (6F-Ins $(1,4,5) \mathrm{P}_{3}$, see Fig. 1), and 1,5-anhydroxylitol 2,3,4-tris(phosphate) (Xylo(1,2,6) $\mathrm{P}_{3}$, see Fig. 2) [8][9]. Both molecules have three ionizable phos-

Fig. 1. Microscopic protonation mechanism of 6-desoxy-6-fluoromyo-inositol 1,4,5-tris(phosphate) $\left(6 \mathrm{~F}-\operatorname{lns}(1,4,5) \mathrm{P}_{3}\right)$ in $0.2 \mathrm{M} \mathrm{KCl}$. (a) Site-specific titration curves derived from ${ }^{31} \mathrm{P}-\mathrm{NMR}$ titration data (points) with best fits of the site-binding model (solid lines). The site in question is indicated by a star and the corresponding number. (b) Macrostate probabilities. (c) Microstate probabilities and microconstants. The filled circles indicate the protonated sites, while the open circles deprotonated ones. phate groups, and their individual site-titration curves have been measured with ${ }^{31} \mathrm{P}$ NMR in $0.2 \mathrm{M} \mathrm{KCl}$ as described above.

Consider first $6 \mathrm{~F}-\mathrm{Ins}(1,4,5) \mathrm{P}_{3}$ as shown in Fig. 1 [8]. This example is chosen to illustrate the effect of the distance between the ionizable groups. The molecular structure suggests that the proximal groups 4 and 5 should interact very strongly. The distant group 1 should be weakly influenced by the other two and behave almost independently; in fact the fluorine substituent strengthens this phenomenon further [8]. The experimentally observed site titration curves shown in Fig. 1a support this conclusion.
Group 1 shows a monophasic titration curve as familiar from a monoprotic acid or base, while the titration curves of groups 4 and 5 show a biphasic behavior. This structure is characteristic for two strongly interacting groups.

The fit of the NMR data with the sitebinding model is shown in Fig. 1a. One can obtain a satisfactory description of the data by making the assumption that the interaction parameters $\varepsilon_{i j}$ are negligible when involving the group 1, namely, by considering this group to be fully independent. Only the interaction parameter between groups 4 and 5 must be fitted, and one ob-
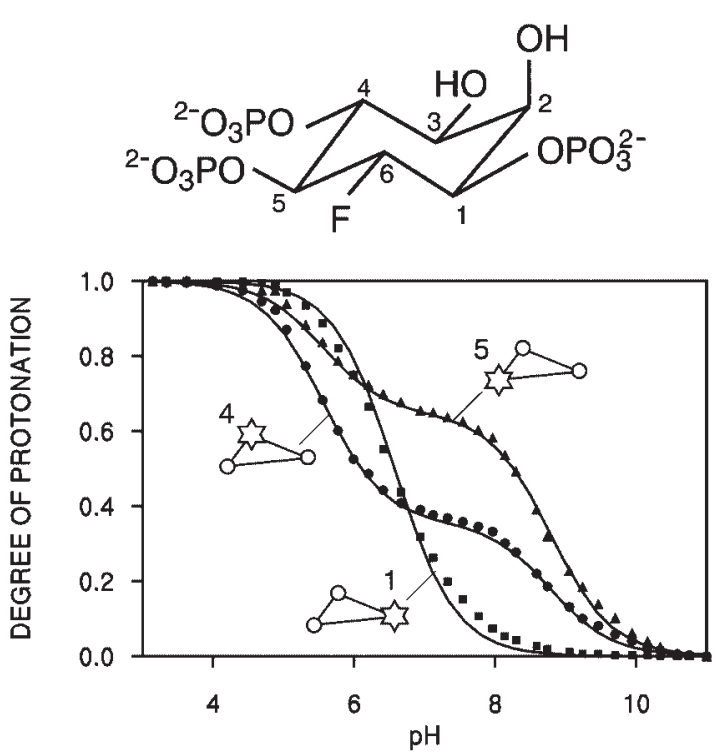

(a)

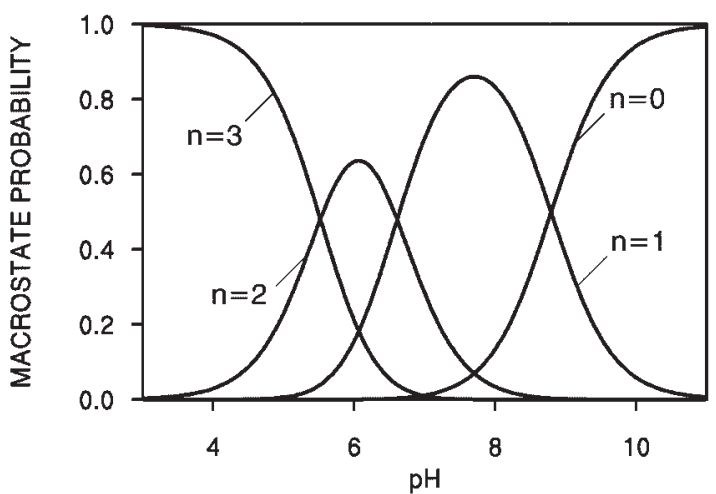

(b)

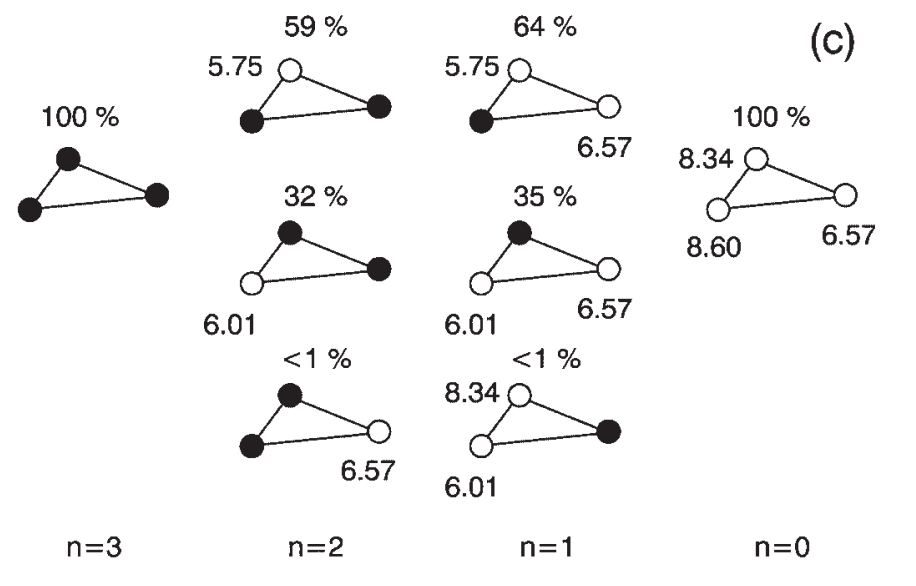


tains 2.59. The corresponding microscopic ionization constants $\mathrm{p} \hat{K}_{i}$ are 6.57, 8.34, and 8.60 for groups 1,4 , and 5, respectively. These values turn out to be all different, since each of these groups has a different chemical environment. One could improve the fit slightly by including additional interaction parameters, but for the sake of simplicity let us proceed with this result. Note that only four adjustable cluster parameters are needed.

With these cluster parameters at hand, we can calculate the macroscopic constants $p K_{n}$. They turn out to be $8.79,6.58$, and 5.52. These constants are accessible from potentiometric titrations, but at this point, their independent determination is only necessary for verification [7][8]. From these constants the macrostate probabilities follow, and they are shown in Fig. 1b. The corresponding conditional probabilities and the microscopic ionization constants are given in Fig. 1c.

The microscopic protonation mechanism of $6 \mathrm{~F}-\operatorname{Ins}(1,4,5) \mathrm{P}_{3}$ can be now described as follows. When decreasing the $\mathrm{pH}$, the group 5 protonates with a microscopic p $\hat{K}$ value of 8.60, while the group 4 with a $\mathrm{p} \hat{K}$ of 8.34 . This leads to two different singly protonated microspecies, the dominant one of $64 \%$ with group 5 protonated, while to a minor one of $35 \%$ with group 4 protonated. These populations are reflected in the site-specific titration curves, which show intermediate plateaus at these values. The microstate, where group 1 is protonated, does not really exist. In the next protonation step, group 1 protonates with a $\mathrm{p} \hat{K}$ of 6.57 , and leads to two doubly protonated microstates with relative populations similar to the previous ones. In the last step, both microstates are protonated with microscopic $\mathrm{p} \hat{K}$ s of 5.75 and 6.01.

The next example of $\mathrm{Xylo}(1,2,6) \mathrm{P}_{3}$ illustrates the effect of symmetry [9]. As evident from Fig. 2a, groups 2 and 6 are equivalent, and thus only two site-titration curves can be measured. The biphasic titration curve belongs to group 1, while the broad monophasic curves belong to groups 2 and 6 .
The data can be again well modeled with a site-binding model, as shown in Fig. 2a. The interaction parameters $\varepsilon_{i j}$ are now important between all groups. The one involving proximal groups 1 and 2 (and equally 1 and 6 ) becomes 1.13 , while the interaction between the more distant groups 2 and 6 is weaker and turns out to be 0.54 . The ionization constants $\mathrm{p} \hat{K}_{i}$ are 8.00 for group 1, and 7.66 for groups 2 and 6 . The fit could be slightly improved by introducing a triplet interaction. Again, only four adjustable cluster parameters are needed.

From these cluster parameters we calculate the macroscopic constants $p K_{n}$ of
$8.28,6.83$, and 5.42, and obtain the macrostate probabilities shown in Fig. 2 b. The microscopic characteristics are shown in Fig. 2c, the microscopic protonation mechanism of Xylo(1,2,6) $\mathrm{P}_{3}$ follows.

When decreasing the $\mathrm{pH}$, the groups 1 and 2 (or 6) all protonate almost simultaneously. While the microscopic $\mathrm{p} \hat{K}$ of group 1 of 8.00 is somewhat larger than $\mathrm{p} \hat{K}$ of group 2 of 7.66 , the fact that there are two identical groups make the resulting microstates almost equally likely. The next protonation step can be thought as simultaneous protonation of these two microspecies. The symmetrical species becomes
Fig. 2. Microscopic protonation mechanism of 1,5-anhydroxylitol 2,3,4-tris(phosphate) $\left(X y l o(1,2,6) \mathrm{P}_{3}\right)$ in $0.2 \mathrm{M} \mathrm{KCl}$. (a) Site-specific titration curves derived from ${ }^{31} \mathrm{P}-\mathrm{NMR}$ titration data (points) with best fits of the site-binding model (solid lines). (b) Macrostate probabilities. (c) Microstate probabilities and microconstants. The symbols have the same meaning as in Fig. 1.

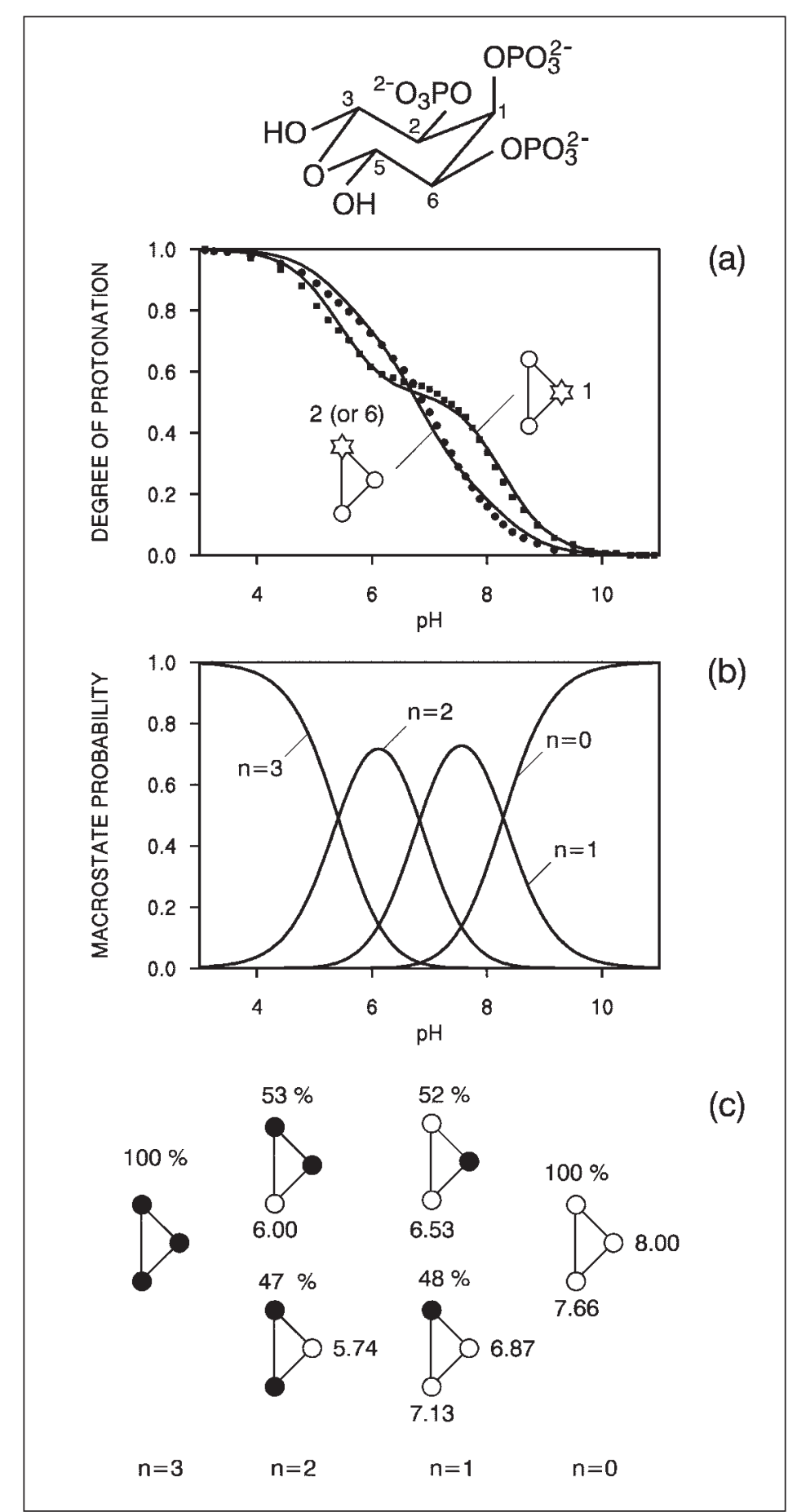


asymmetrical by binding a further proton to group 2 with $\mathrm{p} \hat{K}$ of 6.53 . During this step, group 1 remains protonated, and thus leads to the plateau in its site-specific titration curve. In the other case, the asymmetrical species becomes symmetrical when group 1 with $p \hat{K}$ of 7.13 is being protonated.

\section{Microscopic Equilibria from Potentiometric Titrations}

The common wisdom is that information about microscopic equilibria cannot be extracted from macroscopic potentiometric titration curves. This observation follows from the fact that the macroscopic titration curve only depends on the macroconstants, and the microconstants do not enter.

However, given the alternative parameterization of the problem proposed here, this conclusion must be revisited. All macroconstants (as well as the microconstants) can be expressed in terms of the cluster parameters. Thus, given the macroconstants for a molecule, one can attempt to solve the inverse problem. However, the necessary number of cluster parameters normally exceeds the number of macroconstants, and the solution remains underdetermined. For example, for both triphosphates (with three macroconstants) discussed above, four cluster parameters were needed to describe the situation.

This problem can be overcome, however, by analyzing a homologous series of molecules. To an excellent approximation, the cluster parameters remain the same within such a series. Thus, one can combine the macroconstants for several members of

Table. Comparison of experimental and fitted values of the macroconstants for a homologous series of linear polyamines. the series into a single data set, and find a common set of cluster parameters describing the entire homologous series by means of a least-squares procedure. Already relatively few members in the homologous series can be sufficient to make the solution overdetermined, and the validity of the model can be tested as well. Thus, microconstants can be reliably extracted from macroconstants within such a series. Equivalently, once can obtain the cluster parameters from potentiometric titration curves, but as the macroconstants are usually being tabulated, we prefer to base our analysis on those.

Let us illustrate the procedure with the analysis of linear polyamines with $-\mathrm{NH}-\mathrm{CH}_{2}-\mathrm{CH}_{2}-$ as the repeating unit. The experimentally determined macroconstants in $0.1 \mathrm{M} \mathrm{KCl}$ of the first four members of the series are summarized in the Table [15]. The simplest model assumes two different microconstants $\mathrm{p} \hat{K}^{(\mathrm{I})}$ and $\mathrm{p} \hat{K}^{\text {(II) }}$ for the primary and secondary amines, and nearest neighbor pair interactions with a strength characterized with interaction parameter $\varepsilon$. The best fit of the first three members of the series is shown in the left column, and one observes that the data are not described very well. The next step to improve the model is to include next nearest neighbor pair interaction or nearest neighbor triplet interactions. Least squares fit of the data shows that next nearest neighbor pair interactions are negligible, but that the triplet interactions turn out to be important. The model which includes these interactions provides an excellent fit of the data [11]. The resulting cluster parameters are $\mathrm{p} \hat{K}^{(\mathrm{I})}=$ $9.42, \mathrm{p} \hat{K}^{(\mathrm{II})}=8.44, \varepsilon=1.97$, and $\lambda=0.42$.
The power of the present approach becomes now fully apparent, as only four cluster parameters are needed to describe all equilibria within the entire series.

The model can be further tested by calculating the macroconstants of the tetren. The results are given in the Table. While the model with only nearest neighbor pair interactions fails to reproduce its macroconstants properly, the model including triplet interactions does an excellent job in predicting these values. The model with four cluster parameters thus describes the experimental data in the homologous series very well, and can be used to obtain all other properties with confidence. The pertinent results are summarized in Figs. 3-5.

Fig. 3 shows the situation for dien. The site-specific titration curves are shown in Fig. 3a. One observes that only the primary amine groups protonate at high $\mathrm{pH}$, while the secondary amine mainly protonates at much lower $\mathrm{pH}$. The macrostate probabilities are shown in Fig. 3b, and the splitting of the macrostates in the corresponding microstates is illustrated in Fig. 3c.

The protonation mechanism can be thus described as follows. When one decreases the $\mathrm{pH}$, both primary amines protonate at first in a two-step process. The microspecies with both primary amines protonated is very stable and dominates the picture over a wide $\mathrm{pH}$ range. Only at substantially lower $\mathrm{pH}$ the secondary amine is being protonated. The secondary amine also protonates in an intermediate fashion at higher $\mathrm{pH}$ to a small extent leading to the small peak in the site-specific titration curve.

\begin{tabular}{|c|c|c|c|c|}
\hline Molecule & $N$ & Experimental $^{\mathrm{a}}$ & $\begin{array}{l}p K_{n} \\
\text { Model Ib }\end{array}$ & Model II \\
\hline Ethylenediamine (en) & \multirow[t]{2}{*}{2} & 9.89 & 9.77 & 9.72 \\
\hline $\mathrm{H}_{2} \mathrm{~N}\left(\mathrm{CH}_{2}\right)_{2} \mathrm{NH}_{2}$ & & 7.08 & 6.97 & 7.15 \\
\hline 1,4,7-Triazaheptane (dien) & \multirow[t]{2}{*}{3} & 9.84 & 9.79 & 9.74 \\
\hline $\mathrm{H}_{2} \mathrm{~N}\left(\mathrm{CH}_{2}\right)_{2} \mathrm{NH}\left(\mathrm{CH}_{2}\right)_{2} \mathrm{NH}_{2}$ & & $\begin{array}{l}9.02 \\
4.23\end{array}$ & $\begin{array}{l}9.15 \\
3.98\end{array}$ & $\begin{array}{l}9.10 \\
4.08\end{array}$ \\
\hline 1,4,7,10-Tetrazadecane (trien) & \multirow[t]{4}{*}{4} & 9.74 & 9.80 & 9.76 \\
\hline \multirow{3}{*}{$\mathrm{H}_{2} \mathrm{~N}\left[\left(\mathrm{CH}_{2}\right)_{2} \mathrm{NH}\right]_{2}\left(\mathrm{CH}_{2}\right)_{2} \mathrm{NH}_{2}$} & & 9.07 & 9.20 & 9.16 \\
\hline & & 6.59 & 6.42 & 6.69 \\
\hline & & 3.27 & 3.68 & 3.36 \\
\hline 1,4,7,10,13-Pentazatridecane (tetren) & \multirow[t]{5}{*}{5} & 9.74 & 9.82 & 9.78 \\
\hline \multirow{4}{*}{$\mathrm{H}_{2} \mathrm{~N}\left[\left(\mathrm{CH}_{2}\right)_{2} \mathrm{NH}\right]_{3}\left(\mathrm{CH}_{2}\right)_{2} \mathrm{NH}_{2}$} & & 9.14 & 9.24 & 9.21 \\
\hline & & 8.05 & 8.26 & 8.30 \\
\hline & & 4.70 & 4.45 & 4.73 \\
\hline & & 2.97 & 3.50 & 2.99 \\
\hline
\end{tabular}

aAt an ionic strength of $0.1 \mathrm{M}$. The values are taken from [15].

${ }^{b}$ Best fit with two different models [11]. Model I involves nearest neighbor pair interactions only, while in model II nearest neighbor triplet interactions are included as well. The best fit values for model II are $\mathrm{p} \hat{K}^{(\mathrm{II})}=9.42, \mathrm{p} \hat{K}^{(\mathrm{II})}=8.44, \varepsilon=1.97$, and $\lambda=0.42$. 


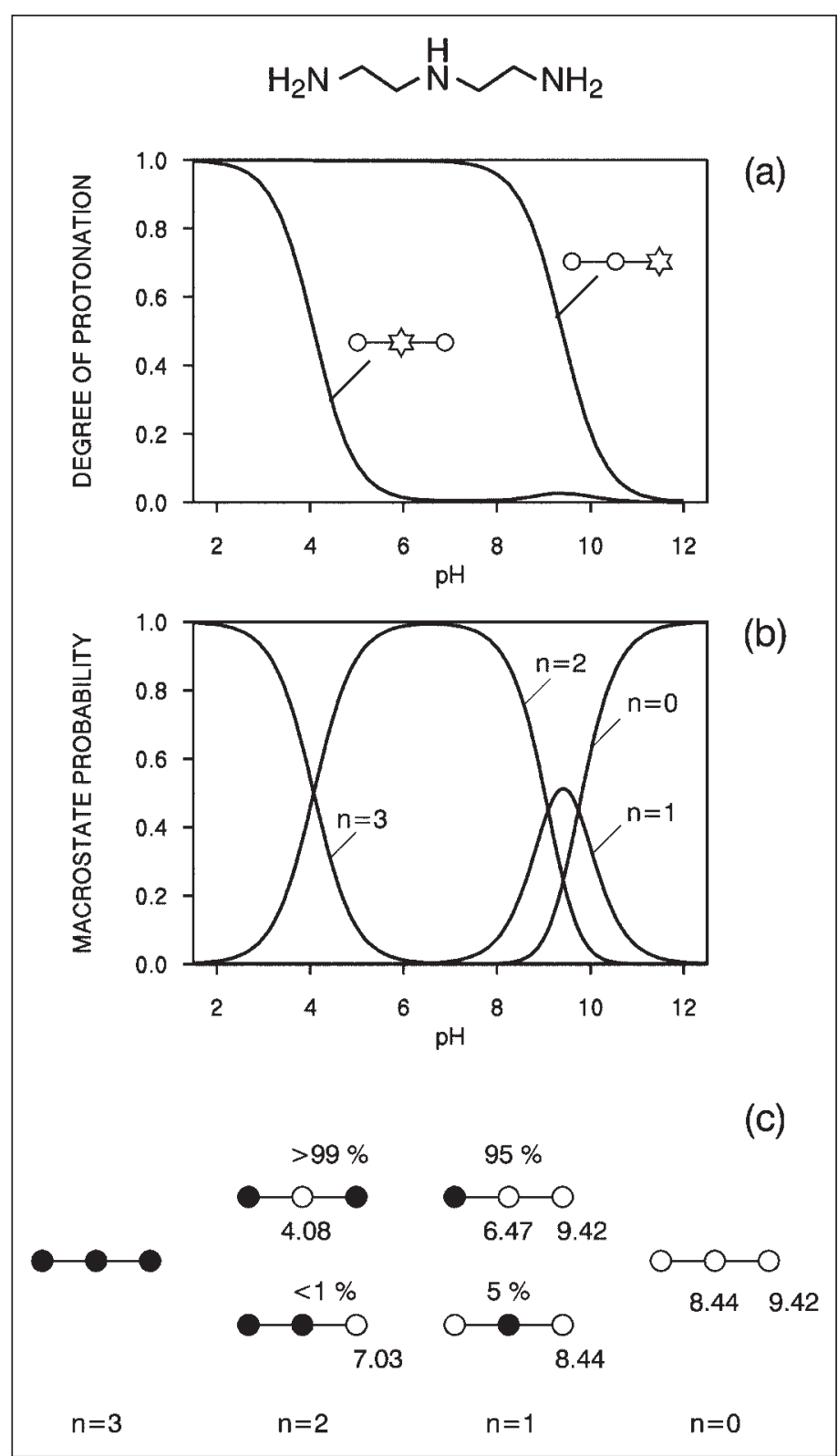

Fig. 3. Microscopic protonation mechanism of 1,4,7-triazaheptane (dien) at $0.1 \mathrm{M} \mathrm{KCl}$ derived from potentiometric titration data (see Table). (a) Calculated site-specific titration curves for the sites labeled by a star. (b) Macrostate probabilities. (c) Microstate probabilities and microconstants. The filled circles indicate the protonated sites, while the open circles deprotonated ones.

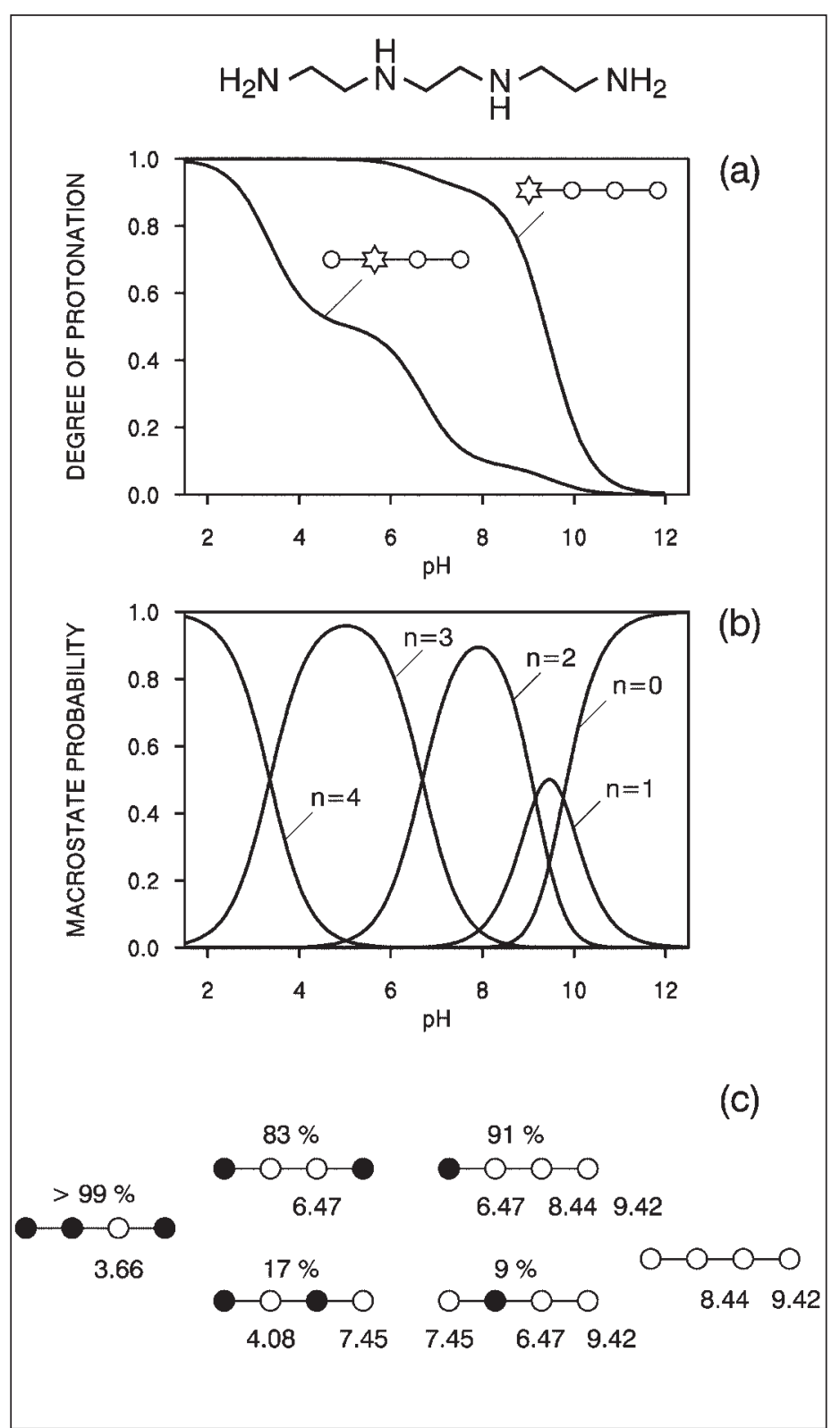

Fig. 4. Microscopic protonation mechanism of 1,4,7,10-tetraazadecane (trien) at $0.1 \mathrm{M} \mathrm{KCl}$ derived from potentiometric titration data (see Table). (a) Calculated site-specific titration curves. (b) Macrostate probabilities. (c) Microstate probabilities and microconstants. Only the most probable states are shown. The fully protonated state is omitted for brevity. The symbols have the same meaning as in Fig. 1.
Fig. 4 illustrates the situation for trien. The site-specific titration curves shown in Fig. 4a now display a more complicated behavior, but reflect a similar pattern to the previous case. The primary amines protonate at high $\mathrm{pH}$, while the secondary amines protonate at lower $\mathrm{pH}$, however, in a twostep fashion in this case. This behavior can be understood by investigating the macrostate probabilities shown in Fig. $4 b$ and the corresponding microstates shown in Fig. 4c. In the first two protonation steps, the protonation of the primary amines is the dominating process, albeit the secondary amines do contribute to a small degree as well. The third and fourth protonation step, the protonation of the secondary amines dominate the picture, which takes place in two distinct steps.

Fig. 5 indicates the corresponding pattern for tetren. In the first protonation step, the protonation of primary amine is most likely, as the secondary groups are more acidic and thus protonate to a lesser extent. During the second step, the other primary group protonates for the same reason. The protonation of secondary groups occurs to a minor extent, as evident from the interme- diate maximum in the site-specific titration curve. The third protonation step leads to the highly symmetric microstate, where both primary and the central secondary groups are protonated. For the fourth protonation step, the prominent microstate is again a symmetric one, where the central secondary amine is deprotonated. The relative stability of this microstate leads to a pronounced deprotonation of the central secondary amine around $\mathrm{pH} 4$.

Further confidence in the present approach can be gained by comparing the present results with the analysis of 


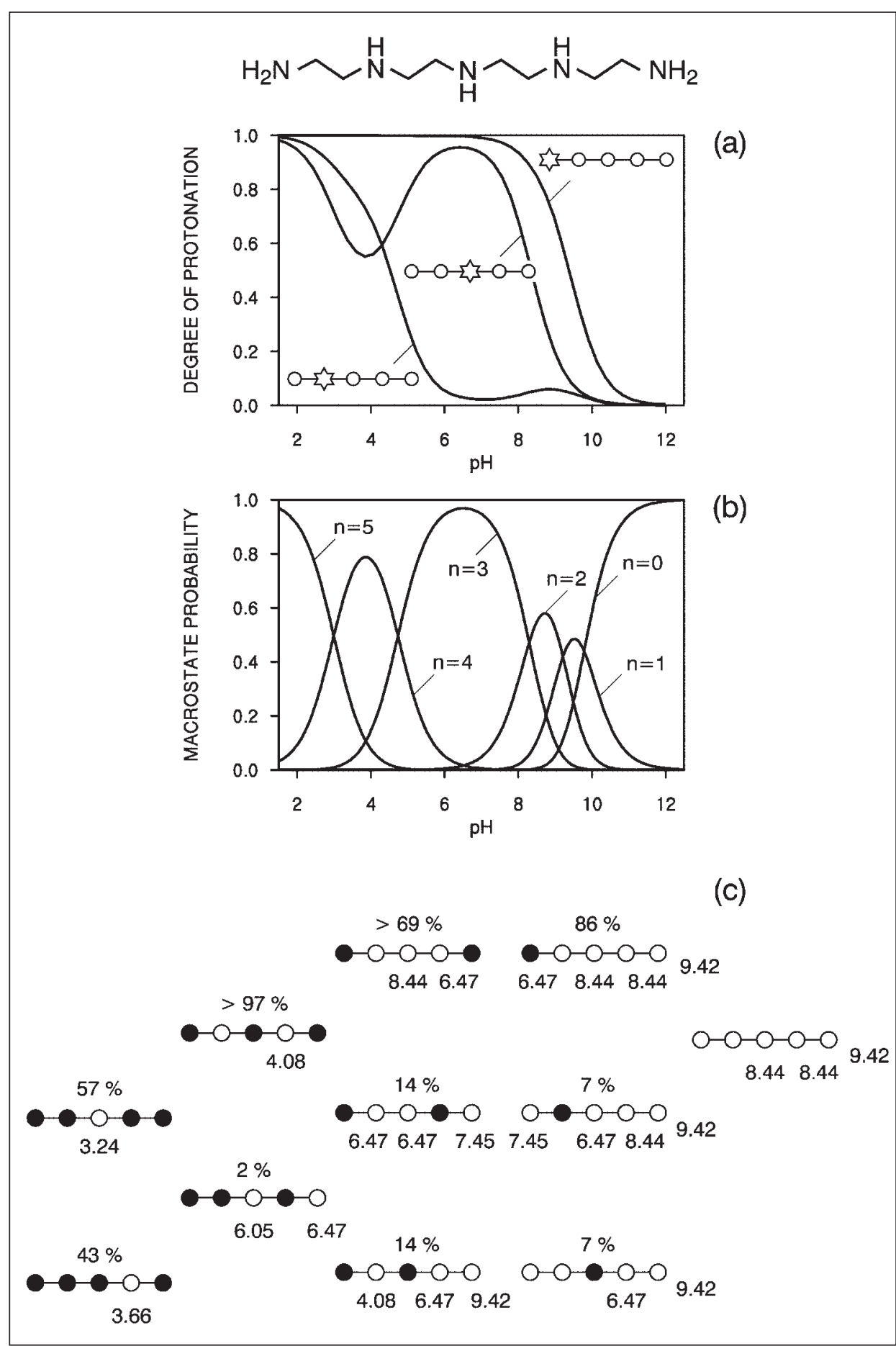

${ }^{13} \mathrm{C}-\mathrm{NMR}$ titration data of tetren [13]. While the medium was rather different, a rather comparable set of cluster parameters was obtained, and basically the same microscopic protonation mechanism was derived.

\section{Conclusion}

We have shown that a novel site-binding model, which is based on a cluster expansion procedure borrowed from statistical mechanics, provides an extremely convenient parameterization of microscopic ionization equilibria. Since these interactions are typically short-ranged, a small set of parameters usually proves sufficient to parameterize the microscopic equilibria properly. This parameterization has substantial advantages over the commonly whose number becomes huge even for moderately sized molecules.

We have presented two practical methods to obtain such cluster parameters. The first approach is based on NMR titration data, where one follows the chemical shifts of spin $1 / 2$ nuclei vicinal to the ionizable groups as a function of $\mathrm{pH}$. The second apused microscopic equilibrium constants,
Fig. 5. Microscopic protonation mechanism of 1,4,7,10,13-pentazadecane (tetren) at $0.1 \mathrm{M}$ $\mathrm{KCl}$ derived from potentiometric titration data (see Table). (a) Calculated site-specific titration curves. (b) Macrostate probabilities. (c) Microstate probabilities and microconstants. Only the most probable states are shown. The fully protonated state is omitted for brevity. The symbols have the same meaning as in Fig. 3.

proach is based on the analysis of the macroconstants within a homologous series of polyprotic molecules. In both cases, one extracts the cluster parameters by means of a least-squares procedure. Once these parameters are known, all properties of the microscopic equilibria can be calculated.

\section{Acknowledgement}

Financial support from Swiss National Science Foundation is gratefully acknowledged (Grants 2100-066514.01 and 8220-067593.01).

Received: September 26, 2002

[1] E.J. King, 'Acid-Base Equilibria', Pergamon Press, Oxford, 1965.

[2] A.E. Martell, R.J. Motekaitis, 'The Determination and Use of Stability Constants', VCH Publishers, New York, 1988.

[3] M. Borkovec, B. Jönsson, G.J.M. Koper, in 'Surface and Colloid Science', Ed. E. Matijevic, vol. 16, Kluwer Academic/ Plenum Publishers, New York, 2001.

[4] J.T. Edsall, R.B. Martin, B.R. Hollingworth, Proc. Natl. Acad. Sci. USA, 1958, 44, 505.

[5] T. Ishimitsu, S. Hirose, H. Sakurai, Talanta 1977, 24, 555-560.

[6] C.P. Schlichter, 'Principles of Magnetic Resonance', Springer, Berlin, 1980.

[7] K. Mernissi-Arifi, L. Schmitt, G. Schlewer, B. Spiess, Anal. Chem. 1995, 67, 2567-2574.

[8] M. Felemez, S. Ballereau, G. Schlewer, B. Spiess, New. J. Chem. 2000, 24, 631-638.

[9] K. Mernissi-Arifi, G. Schlewer, B. Spiess, Carbohydrate Res. 1998, 308, 9-17.

[10] G.J.M. Koper, M.H.P. van Genderen, C. Elissen-Roman, M.W.P.L. Baars, E.J. Meier, M. Borkovec, J. Am. Chem. Soc. 1997, 119, 6512-6521.

[11] M. Borkovec, G.J.M. Koper, J. Phys. Chem. 1994, 98, 6038-6045.

[12] H.S. Robertson, 'Statistical Thermophysics', Prentice Hall, New Jersey, 1993.

[13] M. Borkovec, G.J.M. Koper, Anal. Chem. 2000, 72, 3272-3279.

[14] D.D. Perrin, B. Dempsey, E.P. Serjeant, $\mathrm{pK}_{\mathrm{a}}$ Prediction for Organic Acids and Bases', Chapman \& Hall, London, 1981.

[15] R.M. Smith, A.E. Martell, 'Critical Stability Constants', Plenum Press, New York, vol 6, 1989. 